\title{
\$5. Ordered semigroup strings with restrictions
}

Let $G$ be a nonnegative ordered additive abelian semigroup, let $O$ be a nonnegative integer, and let $u \in G$. We define

$$
\begin{aligned}
& G(0=u)=\{i \in G(0): \operatorname{abs}(i)=u\} \\
& G(0 \geq u)=\{i \in G(0): \operatorname{abs}(i) \geq u\} \\
& G(0>u)=\{i \in G(0): \operatorname{abs}(i)>u\} \\
& G(0<u)=\{i \in G(0): \operatorname{abs}(i)<u\} \\
& G(0 \leq u)=\{i \in G(0): \operatorname{abs}(i) \leq u\} .
\end{aligned}
$$

Given any $P \in\{=, \geq,>,<, s\}$ and any string-restriction $t$, we define

$$
G(O P u, t)=G(O P u) \cap G(o, t)
$$

and for any string-restriction $k$ we define

$$
G(o P u, t, k)=G(o P u) \cap G(o, t, k) \text {. }
$$

